\title{
Results of a Patient-Directed Survey on Frequency of Family History of Glaucoma in 2170 Patients
}

\author{
Gwendolyn Gramer, ${ }^{1}$ Bernhard H. F. Weber, ${ }^{2}$ and Eugen Gramer ${ }^{3}$ \\ ${ }^{1}$ Centre for Paediatric and Adolescent Medicine, Division for Metabolic Disorders, University of Heidelberg, Germany \\ ${ }^{2}$ Institute of Human Genetics, University of Regensburg, Germany \\ ${ }^{3}$ University Eye Hospital, University of Wuerzburg, Germany
}

Correspondence: Gwendolyn

Gramer, Centre for Paediatric and

Adolescent Medicine, Department of General Paediatrics, Division for

Metabolic Disorders, Im Neuenheimer Feld 430, 69120 Heidelberg Germany;

gwendolyn.gramer@med.

uni-heidelberg.de.

Submitted: August 9, 2013

Accepted: November 22, 2013

Citation: Gramer G, Weber BHF, Gramer E. Results of a patient-directed survey on frequency of family history of glaucoma in 2170 patients. Invest Ophthalmol Vis Sci. 2014;55:259264. DOI:10.1167/iovs.13-13020
Purpose. To evaluate in different types of glaucoma frequency of family history of glaucoma (FHG), age at diagnosis, glaucoma risk in relatives, and acceptance rate of genetic glaucoma tests. To assess stage of visual field loss (VFL) in relation to FHG.

MethoDs. Using standardized questions whether an ophthalmologist had found or excluded glaucoma or ocular hypertension $(\mathrm{OH}), 2170$ patients with glaucoma or $\mathrm{OH}$ interviewed all their first and second degree relatives. One thousand three hundred thirty-eight patients had POAG, 233 primary angle closure glaucoma (PACG), $148 \mathrm{OH}, 153$ normal tension glaucoma (NTG), 50 pigmentary glaucoma (PG), and 66 pseudoexfoliation glaucoma (PEX).

Results. Frequency of FHG was 40\% in POAG, without significant differences compared with NTG $(P=0.08)$, OH $(P=0.5)$, PACG $(P=0.4)$, and PG $(P=0.6)$. There were significant differences in age at diagnosis between the glaucomas (smallest between group $P<0.0001$ ). Patients with FHG were significantly younger at diagnosis than patients without FHG in all types of glaucoma (all $P$ values $\leq 0.03$ ), except NTG and PEX. Patients' siblings and mothers had the highest detection probability for glaucoma in POAG and $\mathrm{OH}$. There was no significant relation between stage of VFL and FHG in POAG $(P=0.6)$. Sixty-eight percent of patients would participate in genetic glaucoma tests.

Conclusions. There is a similarly high genetic disposition in all types of glaucoma. Disease risk was especially high in mothers and siblings. In patients with FHG, knowledge of genetic disposition of the glaucomas may have led to earlier diagnosis. This highlights the need for glaucoma awareness campaigns.

Keywords: glaucoma, screening, family history of glaucoma, genetic disposition, risk factors
G laucoma is a multifactorial condition characterized by a progressive optic neuropathy and distinctive visual field loss (VFL). Family history of glaucoma (FHG) is considered a major risk factor for the development of glaucomatous disease, which highlights the importance of a genetic background in this group of diseases. So far there is no clinical study comparing the frequency of FHG between different glaucomas following systematic interviews of all first and second degree relatives. The genetic background of glaucoma is highlighted by the exponentially growing number of molecular genetic studies in glaucoma. As in most complex diseases, the exact number of genes involved in the different glaucomas, their individual contribution to the pathogenesis of the disease, and their specific ways of interference remain largely unknown so far. ${ }^{1,2}$ Several genes have been reported to be associated with different types of glaucoma for example, for POAG, myocillin $[M Y O C],{ }^{3}$ optineurin $[O P T N],{ }^{4}$ and WDR36. ${ }^{5,6}$ However, mutations in these genes account only for a small proportion of less than $10 \%$ of POAG cases. ${ }^{7}$ For many candidate genes results could not be replicated in different populations. ${ }^{7}$ Recent genome-wide association studies (GWAS) identified several common variants associated with POAG, including single nucleotide polymorphisms (SNP) near the caveolin 1 and 2 genes $(C A V 1 / C A V 2)$ in a cohort from Iceland, ${ }^{8}$ and in CDKN2BAS and SIX1/SIX6 regions in patients of European ancestry. ${ }^{9}$ Another GWAS showed a significant association of IOP and SNPs in TMCO1 and GAS $7 .^{10}$

For normal tension glaucoma (NTG) a robust association was found with $C D K N 2 B A S$ variants. ${ }^{9}$ For pseudoexfoliation glaucoma (PEX) it was found that individuals with a specific sequence variation in the $L O X L 1$ gene have an increased risk to develop PEX glaucoma up to 700 -fold higher than individuals without this sequence variant. ${ }^{11}$ The polymorphisms in the LOXL1 gene are specifically associated with PEX, but not with NTG and pigmentary glaucoma (PG). ${ }^{12}$ In addition, significant associations between PEX and SNPs in the CLU and CNTNAP2 genes were found, suggesting that genetic variation in these genes is an additional risk factor for PEX. ${ }^{13,14}$ Despite all new findings about the molecular genetic basis of glaucoma, clinical studies remain essential to provide new insights into clinical characteristics of different glaucomas and disease risk in relatives.

Cross-sectional studies have suggested that FHG conveys an up to 3-fold increased risk to develop POAG. ${ }^{15,16}$ This emphasizes that a thorough family history is crucial for glaucoma screening as it helps to identify people at risk. Therefore, the frequency of FHG and its significance for early diagnosis of glaucoma were evaluated in this study with five purposes: prospective evaluation of (1) differences in the frequency of FHG in patients with POAG, primary angle closure 
TABLE 1. Frequency of FHG in Patients With Different Types of Glaucoma Compared With POAG

\begin{tabular}{lccccc}
\hline Type of Glaucoma & Patients & & \multicolumn{2}{c}{ Patients With FHG } & $\begin{array}{c}\text { Fisher's } \\
\text { Exact Test } \\
\text { (2-Sided) }\end{array}$ \\
\hline POAG & $\boldsymbol{n}$ & & $\boldsymbol{n}$ & $\%$ & \\
PACG & 1335 & & 548 & 41.0 & \\
OH & 233 & & 102 & 43.8 & $P=0.4$ \\
NTG & 148 & & 56 & 37.8 & $P=0.5$ \\
PG & 153 & & 51 & 33.3 & $P=0.08$ \\
PEX & 50 & & 18 & 36.0 & $P=0.6$ \\
& 66 & & 14 & 21.2 & $P=0.001$ \\
\hline
\end{tabular}

glaucoma (PACG), ocular hypertension $(\mathrm{OH})$, NTG, pigmentary glaucoma (PG), and PEX, (2) differences in the age at diagnosis between patients with and without FHG in these different glaucomas, (3) the frequency of patients with POAG, NTG, and $\mathrm{OH}$, in whose relatives (split in 12 groups) glaucoma or $\mathrm{OH}$ was detected or excluded. It was evaluated which group of relatives (e.g., siblings, mothers) had the highest detection probability of glaucoma or $\mathrm{OH}$ in the different glaucomas, (4) the frequency of FHG in POAG patients with no, mild, moderate, and severe VFL. Do patients with FHG show a more severe visual field loss than patients without FHG? (5) The acceptance rate of future genetic tests for glaucoma in glaucoma patients.

\section{MeTHODS}

Two thousand one hundred and seventy glaucoma patients interviewed their siblings, children, parents, and relatives of mother and father (grandmothers, grandfathers, uncles, aunts) by means of a detailed questionnaire ${ }^{17}$ with 16 standardized questions. This central part of the questionnaire resulted in information on FHG in 12 groups of relatives (siblings, children, mother, father, paternal grandmother, paternal grandfather, paternal uncles, paternal aunts, maternal grandmother, maternal grandfather, maternal uncles, maternal aunts) for every patient. The questionnaire used was designed for this study, as no such instrument was available so far. It was developed by the authors and presented to a group of international experts for discussion at the workshop "Genetics in glaucoma." 18 It was agreed upon as a suitable instrument for this study by the experts. The questionnaire was tested in a group of patients in an inpatient setting concerning its feasibility. Patients received instructions in a uniform way by the same glaucoma expert on how to perform the interviews of their relatives before using the questionnaire. Each relative was asked by the patient whether glaucoma or $\mathrm{OH}$ had been diagnosed or excluded by an ophthalmologist. Patients were instructed to read out the questions word by word. Answer categories were yes/no/do not know. Only yes or no answers were included for further evaluation. Family history of glaucoma was defined as glaucoma or $\mathrm{OH}$ in at least one first or second degree relative. Patients were asked to provide information on the total number of relatives in the 12 groups, the number of relatives in the 12 groups in whom glaucoma or $\mathrm{OH}$ had been found or excluded, and the number of relatives in whom no information based on ophthalmological examination was available or who could not be interviewed by the patient. Patients were also asked to provide information on the type of glaucoma in their relatives, but due to lack of reliable answers, this aspect was excluded from further evaluation.

The first part of the questionnaire was always completed by the same glaucoma specialist (EG) at the patient's visit to the University Eye Hospital Wuerzburg concerning the type of glaucoma, age of the patient at diagnosis, and for both eyes stage of VFL and maximal IOP, defined as the highest IOP ever measured. Concerning age at diagnosis, all patients were asked by the same glaucoma specialist (EG) at what age glaucoma had been diagnosed. This was verified for each patient from the medical records. If the answer was uncertain, a resident contacted the patient's ophthalmologist by telephone while the patient was still in the examination room. Thus, age at diagnosis was evaluated in a uniform way and confirmed from multiple sources, if necessary. Many of the patients included were under the care of the same glaucoma specialist (EG) for more than thirty years. This also provides a high reliability of diagnosis (e.g., in NTG).

The glaucoma specialist staged visual fields (VF) in both eyes according to the classification of Aulhorn. ${ }^{19}$ Goldmann perimetry and in addition, wherever feasible, computerized threshold determining perimetry of the central VF were performed. For staging the first available and reliable VF was chosen in order to judge glaucomatous VFL at or close to the time of diagnosis. Visual fields with Aulhorn stages I and II were summarized as mild VFL, stages III and IV as moderate VFL, stage $\mathrm{V}$ as severe VFL, and normal VF as no VFL. The eye with more severe VFL was used for statistical evaluation. The third part of the questionnaire addressed general risk factors (e.g., migraine, Raynaud's phenomenon, heart disease, blood pressure) and systemic medication.

We received 2170 questionnaires suitable for evaluation. We assessed the incidence of FHG in 1338 patients with POAG, 233 with PACG, 148 with OH, 153 with NTG, 50 with PG, and 66 with PEX. Information on age at diagnosis was provided for 1275 patients with POAG, 221 with PACG, 133 with OH, 147 with NTG, 49 with PG, and 65 with PEX. In the POAG group, for example, 1335 patients interviewed 5312 relatives. Results for other types of glaucoma (e.g., primary congenital glauco$\mathrm{ma},{ }^{20}$ glaucoma in patients with Rieger syndrome, ${ }^{21}$ and glaucoma in aniridic patients ${ }^{22}$ ) were not included in this evaluation due to small sample sizes and are published separately. All patients received IOP lowering therapy. The study adhered to the tenets of the Declaration of Helsinki and written informed consent from participants was obtained.

For statistic evaluation $\chi^{2}$ test, Fisher's exact test 2-sided, and Cochran-Armitage trend test were used. A $P$ value less than or equal to 0.05 was considered statistically significant.

\section{RESUlts}

\section{Frequency of FHG in Different Types of Glaucoma}

The overall frequency of FHG in different types of glaucoma is shown in Table 1. Compared with POAG, the frequency of FHG was not significantly different in PACG, OH, NTG, and PG. Only in PEX the frequency of FHG was significantly lower than in POAG (Fisher's exact test, 2-sided $P=0.001$ ).

\section{Age at Diagnosis in Different Types of Glaucoma Compared With POAG}

There was a significant difference in mean age at diagnosis in different types of glaucoma compared with POAG $(54.8 \pm 12.6$ years) for NTG $(57.2 \pm 13.4$ years; $P=0.03), P G(43.8 \pm 13.0$ years; $P<0.0001)$, OH (51.1 \pm 12.0 years; $P=0.001)$, and PEX $(65.5 \pm 7.9$ years; $P<0.0001)$. No significant difference was 
TABLE 2. Differences in Age at Diagnosis in Different Types of Glaucoma Between Patients With and Without Family History of Glaucoma

\begin{tabular}{|c|c|c|c|c|c|c|c|}
\hline $\begin{array}{l}\text { Type of } \\
\text { Glaucoma }\end{array}$ & $\begin{array}{c}\text { Patients } \\
\text { With FHG, } n\end{array}$ & $\begin{array}{c}\text { Mean Age } \\
\text { at Diagnosis, y }\end{array}$ & $\begin{array}{c}\text { Patients } \\
\text { Without FHG, } n\end{array}$ & $\begin{array}{l}\text { Mean Age at } \\
\text { Diagnosis, y }\end{array}$ & $\begin{array}{c}\text { Earlier Diagnosis } \\
\text { in Patients With FHG }\end{array}$ & $\begin{array}{c}\text { Age } \\
\text { Difference, } y\end{array}$ & $P$ Value \\
\hline POAG & 533 & $51.6 \pm 12.8$ & 741 & $57.1 \pm 11.9$ & Yes & -5.5 & 0.0001 \\
\hline PACG & 97 & $52.1 \pm 13.4$ & 124 & $59.2 \pm 11.4$ & Yes & -7.1 & 0.0001 \\
\hline $\mathrm{OH}$ & 53 & $48.3 \pm 13.1$ & 80 & $53.0 \pm 10.9$ & Yes & -4.7 & 0.03 \\
\hline PG & 17 & $35.4 \pm 9.3$ & 32 & $48.2 \pm 12.6$ & Yes & -12.8 & 0.0002 \\
\hline NTG & 50 & $56.8 \pm 13.0$ & 97 & $57.4 \pm 13.7$ & No & -0.6 & 0.8 \\
\hline PEX & 14 & $62.6 \pm 8.0$ & 51 & $66.4 \pm 7.7$ & No & -3.8 & 0.1 \\
\hline
\end{tabular}

found in mean age at diagnosis between POAG and PACG (56.1 \pm 12.8 years; $P=0.16)$.

\section{Differences in the Age at Diagnosis Between Patients With and Without FHG in Different Types of Glaucoma}

Patients with FHG compared with patients without FHG were significantly younger at the time of diagnosis in POAG, PACG, $\mathrm{OH}$, and PG, but not in NTG and PEX (Table 2). Patients with FHG compared with those without FHG were diagnosed 5 years earlier in POAG, 7 years earlier in PACG, 4 years earlier in $\mathrm{OH}$, and almost 13 years earlier in PG.

\section{Frequency of Glaucoma or $\mathrm{OH}$ in 12 Groups of Relatives of Patients With POAG, OH, and NTG}

The frequency of patients, in whose relatives (split in 12 groups of relatives) glaucoma or $\mathrm{OH}$ had been detected or excluded is shown for patients with POAG, NTG, and OH in Table 3. Siblings and mothers showed the highest detection probability for glaucoma or OH in POAG, as well as in OH. In POAG, OH, and NTG female relatives tended to show a higher frequency of glaucoma or $\mathrm{OH}$ than male relatives. Comparing POAG, OH and NTG, family members belonging to the same group of relatives (e.g., mothers) showed a similar relative frequency of glaucoma or $\mathrm{OH}$.

\section{Frequency of FHG in Patients With POAG in Relation to Stage of VFL}

In 1313 POAG patients FHG was found in $41.2 \%$ (256 of 621) of patients without VFL, 40.3\% (166 of 412) of patients with mild VFL, 38.5\% (65 of 169) of patients with moderate VFL, and $46.9 \%$ (52 of 111) of patients with severe VFL. There was no significant relation between stage of VFL and FHG (CochranArmitage trend test, $P=0.6$ ).

\section{Acceptance Rate of Genetic Tests}

Out of 2170 glaucoma patients, $68.5 \%$ would like to have a genetic glaucoma test, while $31.5 \%$ declined.

\section{Discussion}

\section{Family History of Glaucoma in Different Types of Glaucoma}

This is the first study performing a systematic patient-directed survey on the frequency of FHG in all first and second degree relatives in different types of glaucoma, complemented by clinical data provided by the glaucoma specialist. Compared with a population-based study, ${ }^{23}$ in which only $16.1 \%$ of patients with POAG reported FHG, we found a considerably higher proportion of FHG in POAG patients (41\%). The Baltimore Eye Study ${ }^{23}$ evaluated first-degree relatives only, which might explain the lower incidence of FHG. Other studies found a frequency of FHG similar to our study, such as $33.2 \%$ FHG for all types of glaucoma, $37.9 \%$ for POAG, and $35.5 \%$ for $\mathrm{NTG}^{24}$ or even higher frequencies of $\mathrm{FHG}$ in $50 \%$ of POAG patients and $43 \%$ of $\mathrm{OH}$ patients, ${ }^{25}$ or $59.5 \%$ in POAG patients. ${ }^{16}$

The high variability of FHG may partly be explained by the time span between diagnosis of glaucoma and the first interview regarding $\mathrm{FHG}$. In a previous prospective study (Gramer E, IOVS 2000;41:ARVO Abstract 1482), 6031 patients were interviewed on the day of first diagnosis of glaucoma. Of the 4907 patients who were able to provide information on FHG at time of diagnosis, FHG was reported by $18.8 \%$ with $\mathrm{OH}$, $21.1 \%$ with POAG, $13.4 \%$ with NTG, and $16.9 \%$ of patients with other types of glaucoma. In accordance with the present study, these patients did not show significant differences in frequency of FHG between OH, POAG, and NTG. This provides evidence that the incidence of reported FHG presumably depends on duration of the patients' knowledge of the diagnosis, and awareness of the role of genetic factors in glaucoma.

The major limitation of questionnaire-based studies, in particular concerning the topic of FHG, is that the patients' immediate knowledge about FHG in their families is often limited. Patients do not always have the possibility and time to ascertain the actual status of FHG. The advantage of our present study was that patients interviewed all their available relatives systematically with standardized questions, and could therefore provide more exact information on glaucoma or $\mathrm{OH}$ in their relatives. This may also explain the higher frequency of FHG in our study. We cannot exclude that the high frequency of FHG in our study could also be due to the fact that the questionnaire was predominantly completed and returned by patients with FHG (ascertainment bias). However, the fact that the first part of the questionnaire was already filled in by the ophthalmologist at the time of the visit in the glaucoma clinic should have encouraged patients with and without FHG equally to complete the questionnaire. Also, some questions concerned general risk factors, which again should have encouraged patients with and without FHG to complete and return the questionnaire. The high frequency of FHG in our study could also partly be influenced by the fact that at least some patients were referred to the University Eye Hospital because of their FHG, and that consequently patients with FHG might be overrepresented in this glaucoma service (again ascertainment bias). The proportion of affected family members found in our study is much higher than expected according to population prevalence rates, which range from $1.2 \%$ to $5.6 \%$ depending on age and ethnic background. ${ }^{15,26}$ For the evaluation of differences in FHG between the glaucomas, all selection biases discussed above may be irrelevant, as they should influence the different types of glaucoma equally. The limitations of our questionnaire are 
presumably of minor significance as the Glaucoma Inheritance Study in Tasmania revealed FHG in $59.5 \%$ of all patients by direct examination of the relatives of 1700 POAG patients during a 15 -year period. ${ }^{16}$

One strength of this study is that assessment of frequency of FHG and other risk factors was performed in different glaucomas in a uniform way, using the same methods. This approach allows for assessment of differences in FHG and age at diagnosis between the glaucomas. We found no significant differences in the frequency of FHG between different types of glaucoma. This is surprising as molecular genetic studies highlighted differences in genes involved, suggesting genetic heterogeneity in different types of glaucoma. $3,6,9-13,27,28$ In clinical practice, our finding signifies that for all types of glaucoma FHG is equally important and supports the necessity of repeated interviews on FHG in patient care. In the United Kingdom, awareness of the significance of FHG in glaucoma screening already led to implementation of a no cost National Health Service (NHS) sight test for people with the risk factor FHG. ${ }^{29}$

\section{Age at Diagnosis in Different Types of Glaucoma}

Compared with POAG, there was a significant difference in the age at diagnosis between different types of glaucoma. Patients with POAG, PACG, OH, and PG with FHG were significantly younger at the time of diagnosis compared with patients without FHG. In PG, a predominantly binocular disease with early onset and high genetic predisposition, ${ }^{30}$ patients with FHG were diagnosed almost 13 years earlier than those without. We suggest that awareness of FHG, and perhaps a deeper knowledge of the disease, may have led to earlier presentation at the ophthalmologist. In accordance with our results, Landers et al. ${ }^{1}$ found in a study on POAG, that patients with FHG were younger at diagnosis than those without.

Our result that PEX patients with FHG were not significantly younger at diagnosis than those without FHG could be due to the fact that PEX patients are mostly older at disease onset than those with other glaucomas. ${ }^{17}$ At disease onset their relatives might already have passed away, and therefore cannot provide information on glaucoma and raise awareness of the disease, what could have resulted in earlier consultation of an ophthalmologist and might have led to earlier diagnosis. We suggest that the lack of earlier diagnosis in NTG patients with FHG could be explained by the fact that NTG was more frequently diagnosed at a later stage of the disease than IOPdependant glaucomas, also detectable by IOP screening. ${ }^{17}$ In addition, the differences in mean age at diagnosis suggest differences in the onset of the disease in the glaucomas. Knowledge of these differences is important for an effective glaucoma screening.

\section{Disease Risk in Relatives in Different Types of Glaucoma}

Siblings and mothers showed the highest detection probability for glaucoma and $\mathrm{OH}$. Female family members tended to show a higher frequency of glaucoma or $\mathrm{OH}$ than males. In the children of POAG patients no sex difference was found, ${ }^{17}$ but in siblings sisters were more often affected than brothers. ${ }^{17}$ In the Barbados Epidemiological Study one-fifth of 338 siblings of open angle glaucoma (OAG) patients had OAG, but these were more often male. ${ }^{31}$ Overall, approximately one-fourth of the relatives had OAG or were glaucoma suspects. This was found in a population with African heritage, which is also known to be a significant risk factor for OAG. Individuals of African descent generally have a higher prevalence of OAG and tend to develop it at an earlier age. ${ }^{32}$ We suggest that the higher 
frequency of affected sisters in our study may be in part due to an age-related effect and not a sex-related difference. Females have a higher life expectancy and therefore have a higher risk to develop age-related diseases. Whether this age effect fully explains why mothers compared with fathers, and grandmothers compared with grandfathers had a higher frequency of glaucoma or $\mathrm{OH}$ is unknown. The age of the relatives was not asked in the questionnaire. In addition, cultural or social factors may be of relevance.

A study on NTG found an association of SNPs in the genes for Mitofusin 1 and 2, suggesting an involvement of mitochondrial inheritance in NTG. ${ }^{33}$ A further study showed that people with haplogroup $U$ have a lower risk to develop exfoliation glaucoma. ${ }^{34}$ These results suggested that mitochondrial alterations may be involved in the etiology of glaucoma. However, like for many other glaucoma genes, the association of Mitofusin 1 and 2 and NTG has so far not been replicated in other studies. Former studies also showed that in NTG a maternal history of glaucoma is more prevalent than a paternal history. ${ }^{35-37}$ This more pronounced maternal inheritance could support the involvement of mitochondrial dysfunction in glaucoma.

\section{Stage of Visual Field Loss in Relation to FHG in POAG}

In our study, POAG patients with severe VFL did not show a significantly higher frequency of FHG than patients with no, mild, or moderate VFL. This is a comforting result for patients with FHG, who are often concerned about a presumably higher risk of blindness due to their FHG. From our data, we did not find evidence for an increased risk to develop a more severe VFL in patients with FHG, given timely diagnosis and appropriate treatment. Also in other studies, we did not find a significant relation between stage of VFL and FHG in patients with NTG (Gramer E, IOVS 2006; 47:ARVO E-Abstract 3395) and in patients with PG. ${ }^{30} \mathrm{Wu}$ et al. ${ }^{16}$ found, based on a different definition of the stage of the disease than the studies mentioned above, a significant difference in severity of familial glaucoma compared with sporadic glaucoma.

\section{Conclusions}

Our study highlights that glaucoma screening in relatives of glaucoma patients based on clinical examination is very effective in siblings, more effective in females than males, and significant in all types of glaucoma for early detection of the different types of glaucoma. Therefore, awareness campaigns informing the population about the role of genetic factors in the glaucomas are important in order to provide screening examinations for all individuals with FHG. The potential future of genetic testing in glaucoma, which was shown in our study to have a high acceptance rate in glaucoma patients, may hopefully allow to genetically identify family members with or at risk for the disease. This would allow to focus glaucoma screening even more effectively in this highrisk group. Family history of glaucoma is a fate, because relatives have an increased risk to develop the same disease, but it also offers the chance for early diagnosis, early treatment, and thus a better prognosis concerning the prevention of irreversible blindness.

\section{Acknowledgments}

The authors thank all patients and their families for participation in this study. They also thank Georg Burkhard and Elmar Beck
(Statistical Institute Anfomed, Möhrendorf) for support with the statistical analysis.

Support for travel expenses to a scientific meeting from Merck Serono (GG), and by a research scholarship (Olympia Morata programme) of the Medical faculty of the University of Heidelberg (GG).

Disclosure: G. Gramer, Merck Serono (R); B.H.F. Weber, None; E. Gramer, None

\section{References}

1. Landers J, Goldberg I, Graham S. Does a family history of glaucoma affect disease severity at the time of diagnosis? $J$ Glaucoma. 2003;12:31-35.

2. Stöhr H, Weber BHF. Genetik der altersabhängigen Makuladegeneration. In: Holz FG, Pauleikhoff D, Spaide RF, Bird AC, eds. Altersabbängige Makuladegeneration. Berlin-Heidelberg, Germany: Springer Verlag; 2004;26-31.

3. Stone EM, Fingert JH, Alward WL, et al. Identification of a gene that causes primary open angle glaucoma. Science. 1997;275: 668-670.

4. Rezaie T, Child A, Hitchings R, et al. Adult-onset primary openangle glaucoma caused by mutations in optineurin. Science. 2002;295:1077-1079.

5. Monemi S, Spaeth G, DaSilva A, et al. Identification of a novel adult-onset primary open-angle glaucoma (POAG) gene on 5q22.1. Hum Mol Genet. 2005;14:725-733.

6. Hauser MA, Allingham RR, Linkroum K, et al. Distribution of WDR36 DNA sequence variants in patients with primary openangle glaucoma. Invest Ophthalmol Vis Sci. 2006;47:25422546.

7. Bettin P, Di Matteo F. Glaucoma: present challenges and future trends. Ophthalmic Res. 2013;50:197-208.

8. Thorleifsson G, Walters GB, Hewitt AW, et al. Common variants near CAV1 and CAV2 are associated with primary open-angle glaucoma. Nat Genet. 2010;42:906-909.

9. Wiggs JL, Yaspan BL, Hauser MA, et al. Common variants at 9 p21 and 8 q22 are associated with increased susceptibility to optic nerve degeneration in glaucoma. PLoS Genet. 2012;8: e1002654.

10. van Koolwijk LM, Ramdas WD, Ikram MK, et al. Common genetic determinants of intraocular pressure and primary open-angle glaucoma. PLoS Genet. 2012;8:e1002611.

11. Thorleifsson G, Magnusson KP, Sulem P, et al. Common sequence variants in the LOXL1 gene confer susceptibility to exfoliation glaucoma. Science. 2007;317:1397-1400.

12. Wolf C, Gramer E, Muller-Myhsok B, et al. Lysyl oxidase-like 1 gene polymorphisms in German patients with normal tension glaucoma, pigmentary glaucoma and exfoliation glaucoma. $J$ Glaucoma. 2010;19:136-141.

13. Krumbiegel M, Pasutto F, Mardin CY, et al. Exploring functional candidate genes for genetic association in german patients with pseudoexfoliation syndrome and pseudoexfoliation glaucoma. Invest Ophthalmol Vis Sci. 2009;50:2796-2801.

14. Krumbiegel M, Pasutto F, Schlotzer-Schrehardt U, et al. Genome-wide association study with DNA pooling identifies variants at CNTNAP2 associated with pseudoexfoliation syndrome. Eur J Human Genet. 2011;19:186-193.

15. Tielsch JM, Sommer A, Katz J, Royall RM, Quigley HA, Javitt J. Racial variations in the prevalence of primary open-angle glaucoma. The Baltimore Eye Survey. JAMA. 1991;266:369374.

16. Wu J, Hewitt AW, Green CM, et al. Disease severity of familial glaucoma compared with sporadic glaucoma. Arch Ophthalmol. 2006;124:950-954.

17. Gramer G. Familienanamnese, genetisches Risikoprofil und Risikofaktoren der Glaukome. Eine Untersuchung von 2170 
Patienten mit Glaukom oder Okulärer Hypertension Available at: http/:www.opus-bayern.de/uni-wuerzburg/volltexte/2006/ 1997. Accessed August 7, 2013.

18. Tausch M. Den Glaukom-Genen auf der Spur. Internationaler Workshop "Genetics in Glaucoma" in Würzburg. Z prakt Augenbeilkd. 1996;448-452.

19. Aulhorn E. Sensoric functional damage. In: Heilmann K, Richardson KT, eds. Glaucoma. Conceptions of a Disease. Stuttgart, Germany: Thieme; 1978;157-168.

20. Weisschuh N, Wolf C, Wissinger B, Gramer E. A clinical and molecular genetic study of German patients with primary congenital glaucoma. Am J Ophthalmol. 2009;147:744-753.

21. Dressler S, Meyer-Marcotty P, Weisschuh N, et al. Dental and craniofacial anomalies associated with Axenfeld-Rieger Syndrome with PITX2 mutation. Case Rep Med. 2010;2010: 621984

22. Gramer E, Reiter C, Gramer G. Glaucoma and frequency of ocular and general diseases in 30 patients with aniridia: a clinical study. Eur J Ophthalmol. 2012;22:104-110.

23. Tielsch JM, Katz J, Sommer A, Quigley HA, Javitt JC. Family history and risk of primary open angle glaucoma. The Baltimore Eye Survey. Arch Ophthalmol. 1994;112:69-73.

24. Budde WM, Mardin CY, Naumann GO. Positive family anamnesis in patients in the "DFG (German Research Group) Glaucoma Clinical Research Group [in German]. Klin Monbl Augenbeilkd. 1995;207:292-294.

25. Shin DH, Becker B, Kolker AE. Family history in primary openangle glaucoma. Arch Opbthalmol. 1977;95:598-600.

26. Kahn HA, Milton RC. Alternative definitions of open-angle glaucoma. Effect on prevalence and associations in the Framingham eye study. Arch Ophthalmol. 1980;98:21722177.

27. Weisschuh N, Neumann D, Wolf C, Wissinger B, Gramer E. Prevalence of myocilin and optineurin sequence variants in
German normal tension glaucoma patients. Mol Vis. 2005;11: 284-287.

28. Ozel AB, Moroi SE, Reed DM, et al. Genome-wide association study and meta-analysis of intraocular pressure [published online ahead of print September 13, 2013]. Hum Genet. doi:10.1007/s00439-013-1349-5.

29. National Health Service. Eye care entitlements. Available at: http://www.nhs.uk/nhsengland/healthcosts/pages/ eyecarecosts.aspx. Accessed March 22, 2013.

30. Gramer E, Thiele H, Ritch R. Family history of glaucoma and risk factors in pigmentary glaucoma. A new clinical study [in German]. Klin Monbl Augenheilkd. 1998;212:454-464.

31. Leske MC, Nemesure B, He Q, Wu SY, Fielding Hejtmancik J, Hennis A. Patterns of open-angle glaucoma in the Barbados Family Study. Ophthalmology. 2001;108:1015-1022.

32. Wilson R, Martone JF. Epidemiology of chronic open-angle glaucoma. In: Ritch R, ed. The Glaucomas. St. Louis, MO: Mosby; 1996;753-768.

33. Wolf C, Gramer E, Muller-Myhsok B, et al. Evaluation of nine candidate genes in patients with normal tension glaucoma: a case control study. BMC Med Genet. 2009;10:91.

34. Wolf C, Gramer E, Muller-Myhsok B, Pasutto F, Wissinger B, Weisschuh N. Mitochondrial haplogroup $U$ is associated with a reduced risk to develop exfoliation glaucoma in the German population. BMC Genet. 2010;11:8.

35. Morgan RW, Drance SM. Chronic open-angle glaucoma and ocular hypertension. An epidemiological study. Br J Ophthalmol. 1975;59:211-215.

36. Charliat G, Jolly D, Blanchard F. Genetic risk factor in primary open-angle glaucoma: a case-control study. Ophthalmic Epidemiol. 1994;1:131-138.

37. Nemesure B, Leske MC, He Q, Mendell N. Analyses of reported family history of glaucoma: a preliminary investigation. The Barbados Eye Study Group. Ophthalmic Epidemiol. 1996;3: 135-141. 